\title{
Viral Metagenomics of Blood Donors and Blood-Derived Products Using Next-Generation Sequencing
}

\author{
Sophie Waldvogel-Abramowski ${ }^{a}$ b Sofiane Taleb ${ }^{c}$ Marco Alessandrini ${ }^{d}$ \\ Olivier Preynat-Seauve $e^{e, f}$ \\ aLaboratory of Immunohematology, Division of Laboratory Medicine, Department of Diagnostics, Geneva

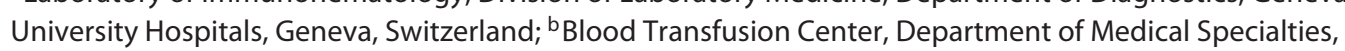

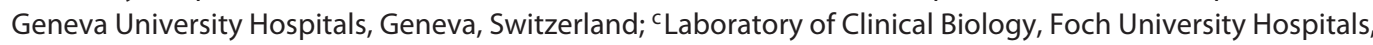 \\ Suresnes, France; ${ }^{\mathrm{d} D e p a r t m e n t}$ of Pathology and Immunology, Faculty of Medicine, University of Geneva, \\ Geneva, Switzerland; ' Laboratory of Therapy and Stem Cells, Department of Diagnostics, Geneva University \\ Hospitals, Geneva, Switzerland; ' Department of Medical Specialties of internal Medicine, Faculty of Medicine, \\ University of Geneva, Geneva, Switzerland
}

\author{
Keywords \\ Blood products - Transfusion-associated infections · Virus \\ safety · Metagenomics · Next-generation sequencing
}

\begin{abstract}
Transfusion-transmitted infections remain a permanent threat in medicine. It keeps the burden of the past, marked by serious infections transmitted by transfusion, and is constantly threatened by emerging viruses. The global rise of immunosuppression among patients undergoing frequent transfusions exacerbates this problem. Over the past decade, criteria for donor selection have become increasingly more stringent. Although routine nucleic acid testing (NAT) for virus-specific detection has become more sensitive, these safety measures are only valuable for a limited number of select viruses. The scientific approach to this is however changing, with the goal of trying to identify infectious agents in donor units as early as possible to mitigate the risk of a clinically relevant infection. To this end, and in addition to an epidemiological surveillance of the general population, researchers are adopting new methods to discover emerging infectious agents, while simultaneously screening for an extended number of viruses in donors. Next-generation sequencing (NGS) offers the opportunity to explore the entire viral landscape in blood donors, the so-called metagenom-
\end{abstract}

ics, to investigate severe transfusion reactions of unknown etiology. In the not too distant future, one could imagine this platform being used for routine testing of donated blood products.

(c) 2019 S. Karger AG, Basel

\section{Nucleic Acid Testing versus Next-Generation Sequencing}

Conventional nucleic acid testing (NAT) detects only a selected sequence of the genome of a virus, with a known and validated sensitivity and specificity. In contrast, nextgeneration sequencing (NGS) has the advantage to cover whole viral genomes without any idea of the sensitivity and specificity of detection of the individual sequences, which in any case may be variable depending on the bioinformatics pipeline used. For example, in a study comparing two pipelines (VirCapSeq-VERT and unbiased NGS), slightly different results were reported [1]. One sequencing method was more sensitive, and the other one provided a better genome coverage. With our existing knowledge, quantitative NAT seems to be more sensitive than an NGS approach. In our study, performed on blood products, the sensitivity of torque teno virus (TTV) detection was higher by conventional NAT than by NGS [2].

\section{KARGER}

(C) 2019 S. Karger AG, Basel 
Nevertheless, NGS should theoretically provide more discriminant results than conventional NAT in case of a viral co-infection or mixed genotypes. Satisfactory performance of this method has been demonstrated with hepatitis $C$ virus mixed-genotype infections [3]. Validation assays and comparative studies are still needed and crucial if NGS is to be implemented for routine diagnostic testing. Anyhow, due to its ability to sequence viral nucleic acid on a very large scale, NGS remains the most suitable tool to detect a biologically significant outbreak. This is a particularly relevant subject in the field of transfusion medicine.

\section{Metagenomics in Human Blood}

\section{General Human Population}

Nonpathogenic viruses are present in the peripheral blood of the general human population. Annelloviruses are the most prevalent family [4], and metagenomics studies have identified a great degree of genetic diversity within this group $[5,6]$, including TTV which are separated into 39 genotypes and infect the majority of humans without any presentation of symptoms. Other viruses found in the blood of the healthy and asymptomatic populations are members of the Picornaviridae, Poxviridae, Flaviviridae, rhabdoviruses and Phycodnaviridae families $[7,8]$. Recently, in a study using NGS to analyze the blood of 8,240 individuals without any symptoms of infectious diseases [4], it was reported that 94 viral species were identified, of which 19 were found in $42 \%$ of participants. A broad representation of herpesviruses and anelloviruses was noticed, along with the identification of seven different types of papillomaviruses (including the oncogenic HPV16), and more or less pathogenic species including human immunodeficiency virus, hepatitis B virus, polyomaviruses and parvovirus B19. The Merkel cell polyomavirus (MCPyV) was detected in $0.55 \%$ of participants [9].

\section{Blood Donors}

Blood donors belong to a particular subpopulation of healthy individuals where the clinical history is compatible with a blood donation. As known for the general population, the anellovirus family is confirmed to be the most prevalent group identified in blood donors [10]. Human pegivirus is also highly represented with an estimated prevalence of $1-5 \%$ in developed countries [11, 12]. Giant blood marseillevirus (GBM) was identified in blood samples from asymptomatic donors [13], with corresponding viral DNA found in $4 \%$ of donors and $9.1 \%$ of a cohort of polytransfused thalassemic patients, suggesting a possible transfusion-mediated transmission [14]. However, two other studies performed on blood donors and polytrans- fused patients did not confirm GBM by NAT $[15,16]$, suggesting that this signature could be a laboratory contaminant rather than a novel virus infecting healthy individuals. On the other hand, DNA from papillomaviruses has been identified by NAT in $8.3 \%$ of healthy male blood donors [17]. Similar to the general population, MCPyV has been reported in $22 \%$ of blood samples from donors by using NAT [9]. To identify new, unexpected or emergent viruses that could be present in the human blood, Sauvage et al. [10] used an ultra-deep sequencing method to analyze samples collected from subjects with an increased risk of infection, including recipients of multiple transfusions. No other sequences suggestive of new viral species were identified in samples with expected commensal viruses.

\section{Metagenomics in Blood-Derived Products}

Our team conducted a metagenomics analysis on samples collected from three main types of blood products manufactured at the blood transfusion center of the University Hospitals of Geneva $[2,18]$. Sequences from common viruses described in healthy individuals and blood donors such as anelloviruses, human pegivirus, $\mathrm{MCPyV}$ and human papillomavirus 27 were detected by NGS in pools made from fresh frozen plasma (FFP), platelet concentrates (PC) and red blood cell units (RBCU). Surprisingly, unexpected sequences from a human astrovirus MLB2 were detected in an FFP and the serum of the corresponding donor, describing a novel asymptomatic infection in donors. Zhang et al. [19] analyzed the viral landscape of large plasma pools dedicated to fractionation, by using an NGS technology. In addition to the expected commensal signatures of anelloviruses and human pegivirus, they detected few sequences of papillomaviruses and two distinct gemycircularvirus genomes. However, several similar genomes have also been identified in environmental samples, plants and animal tissues, opening the possibility of exogenous contamination [20].

\section{Technical Aspects of NGS Applied to Blood Products and Donor}

\section{Which Nucleic Acids Must Be Targeted for a Virus} Screen by NGS?

At their replication phase, DNA viruses produce RNA transcripts, whereas some RNA viruses can also reversetranscribe their genomes to produce DNA. Therefore, DNA extraction plus RNA extraction or a total nucleic acid isolation strategy will be required to ensure exhaustive screening of all existing viruses. Nucleic acids are present in different compartments of blood products 


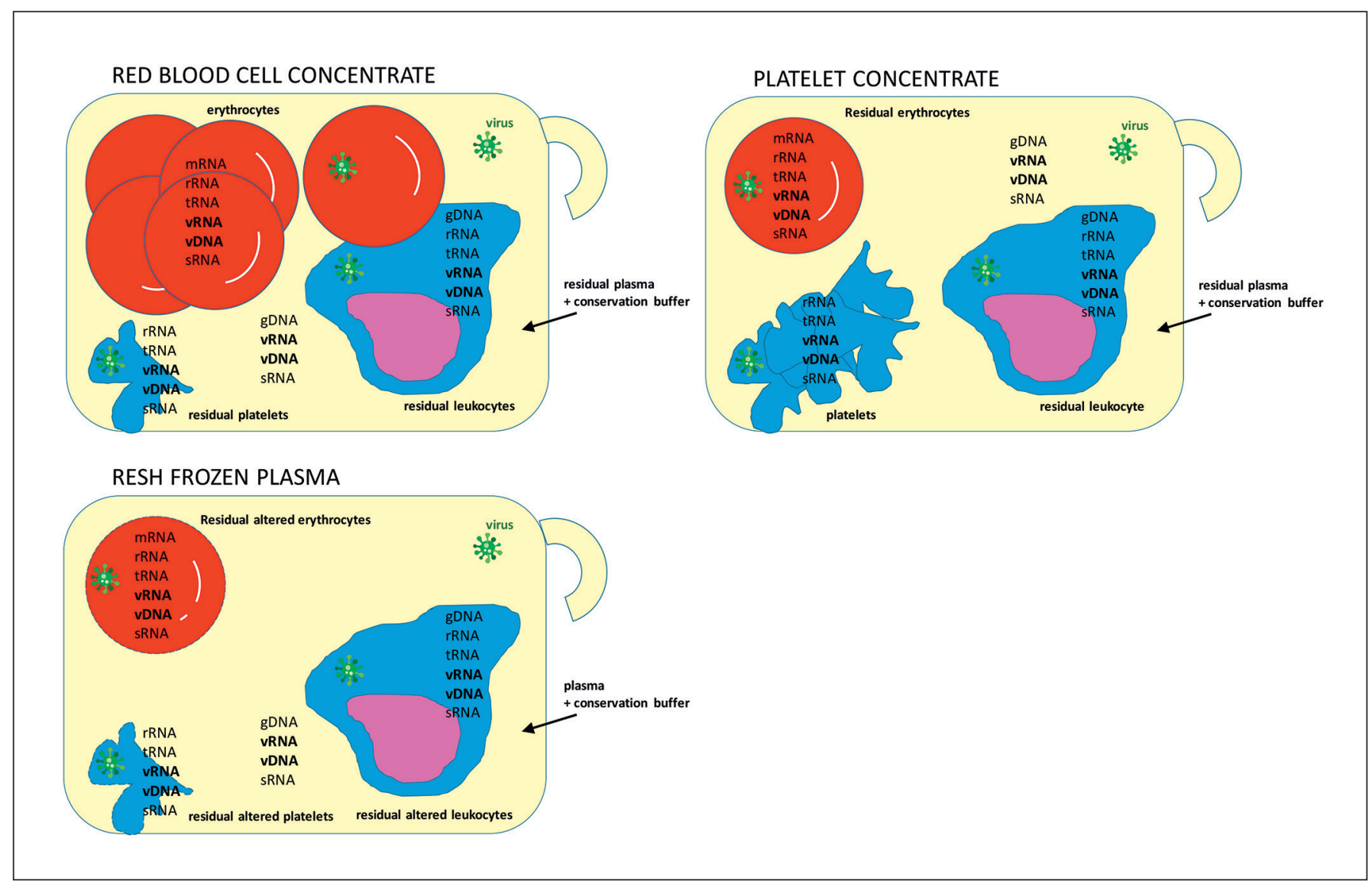

Fig. 1. Nature and localization of nucleic acids in blood products. This scheme represents the various types of nucleic acids found in the different compartments of blood-derived products. The main source is from nucleated cells present within the product. It is mainly from residual leukocytes present in PC, RBCU and FFP. It is noteworthy that during the preparation of FFP, the freezing process results in a release of free or altered nuclei/cells, which in turn alters the residual leukocytes. Within these residual nucleated cells, the entire human genome is present as a double-stranded DNA
(dsDNA), with the possibility of viral dsDNA integration. dsDNA or single-stranded DNA (ssDNA) of viral origin, which is not integrated in the host genome can also be found. In addition to genomic DNA, nucleated cells contain various types of RNAs, including mRNA, tRNA, siRNA, rRNA and small RNAs. DNA viruses at their replication phase will also produce RNA. rRNA, ribosomal RNA; tRNA, transfer RNA; vRNA, viral RNA; sRNA, small RNA; gDNA, genomic DNA; vDNA, viral DNA.

ucts. It is also theoretically possible that, if all of these compounds were previously treated for a total inactivation of pathogens, resting inactive viral nucleic acids could be present. For example, sequences from Kadipiro virus, XMRV and parvovirus-like hybrid genome were described in some laboratory media or reagents [28-30]. Some countries use a pathogen reduction process, such as inactivation by amotosalen, during the manufacturing of products. This process is based on cross-linking of pathogen nucleic acids to impair their replication. It is suggested that nucleic acid cross-linking could theoretically impair the ability to identify viral infection. We have nevertheless observed that a parvovirus B19 in infected FFP, treated by amotosalen, was still detectable in high amounts at a depth similar to untreated products (unpubl. data). These observations suggest that NGS could also be used for viral screening of amotosalen-treated blood products. 
Fig. 2. Interpretation of depth and genome coverage values. A The depth of sequencing indicates how many times a read, i.e. a fragment sequence (nucleic acids are generally fragmented prior to sequencing) attributed to a viral sequence, is detected. Sequence reads with a high depth reflect a high abundance of a virus in the sample. B The second parameter, namely the percentage of genome coverage, refers to the percentage of the viral genome covered by detected reads. C A high percentage of genome coverage is highly suggestive of good specificity and the correct attribution to one defined virus. D In contrast, a low percentage of genome coverage, focused on one fragment of the genome, is more indicative of a misattribution, generally due to homologous sequences of the human genome not being correctly filtered out at the initial stages of data processing.
A

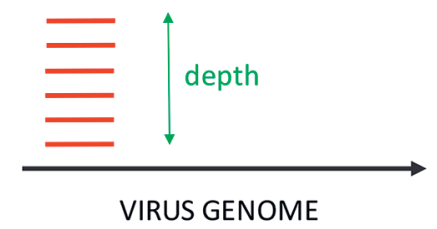

read

C

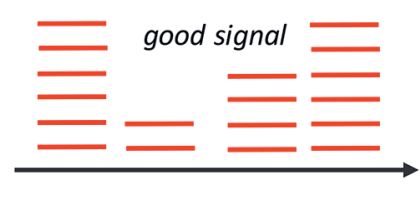

VIRUS GENOME
B

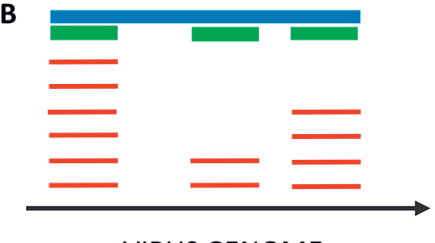

VIRUS GENOME

$\%$ of genome covered $=$ green $/$ blue

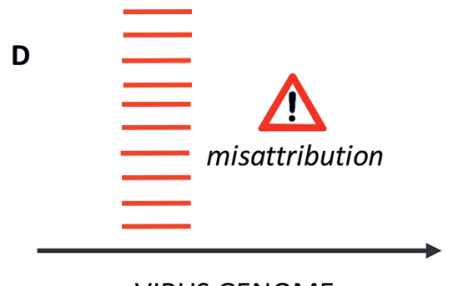

VIRUS GENOME

\section{Methods of Nucleic Acids Isolation from Blood \\ Products}

Numerous methods of nucleic acid extraction are available, all of which are mainly based on the fixation and elution from affinity columns. The biochemical properties of samples influence the yield of extraction, depending on nucleic acid concentration, their nature (DNA or RNA) and the biochemical composition of the sample (acellular, cellular, lipophilic, hydrophilic, etc.). In the case of blood products, the following criteria must be carefully considered when choosing the extraction method: (i) due to a leukocyte reduction prior to manufacturing, total nucleic acids are present at very low concentrations. As a case in point, we observed in FFP a dsDNA concentration ranging from $2.8 \pm 0.9 \mathrm{ng} / \mathrm{mL}$ [27]; (ii) RBCU contain mainly conservation buffer and erythrocytes (hemoglobin); (iii) PC mainly contain residual plasma, conservation buffer and platelets. From our experience, methods designed for the isolation of cell-free nucleic acid from all three types of blood products showed good yields [27].

\section{Preparation of Nucleic Acids for Sequencing}

Prior to sequencing, it is necessary to distinguish DNA viruses from RNA viruses. To achieve this, samples are prepared independently for RNAseq and DNAseq purposes. Samples for RNAseq are prepared by RNA extraction or total nucleic acid extraction, followed by DNA removal using DNAse enzyme. Similarly, samples for DNAseq are prepared via DNA extraction or total nucleic acids extraction, followed by RNA removal using RNAse enzyme. Importantly, some methods such as Illumina or Ion Torrent require double-stranded nucleic acid to enable sequencing. To ensure that any singlestranded viral nucleic acid (RNA or DNA) is not overlooked, it is important and mandatory to convert all single-stranded nucleic acids into a double strand of ssRNA/ cDNA using reverse transcriptase enzymes. Similarly, ssDNA are converted into dsDNA by the use of the Klenow polymerase in the presence of random primer sequences [2]. In the case of RBCU, an abundance of globin mRNA risks competitive saturation of chips used in sequencing. Depletion of globin mRNA is thus recommended to increase the sensitivity of sequencing [2]. Once obtained, the samples can be sequenced by various methods including Illumina technology (www.illumina.com), Ion Torrent sequencing technology (www.thermofisher.com), Oxford nanopore technology (www.nanoporetech.com) or SMRT sequencing (www.pacb.com).

\section{Analysis of Libraries and Data Interpretation}

Library analysis and data interpretation are an important and often challenging endeavor. Human sequences from the host genome, which represent the majority of data in the libraries, should first be removed through mapping with reference databases. Again, special attention should be given to human endogenous retroviral sequences representing a large part of the human genome. Resting genomes are compared with those in different databases of viral genomes, with appropriate filters and algorithms to ensure a correct attribution. It is best to derive a list of detected viral genomes with corresponding probabilistic values of signal pertinence. Two parameters are of particular interest: the depth of sequencing and the percent of genome coverage (Fig. 2). 


\section{Choice of the Bioinformatics Pipeline}

For the choice of the bioinformatics pipeline, several strategies are available: (i) virus discovery-oriented pipelines and (ii) diagnostics-oriented pipelines. The virus discovery-oriented pipelines include generally few filters to remove misattributed signals and unspecific background. They have the advantage of being highly sensitive, allowing for manual removal of false signals with appropriate controls. However, due to the minimal use of filters, they suffer from a lack of specificity, providing long lists of environmental contaminants and consequently time-consuming analysis. Therefore, these pipelines are more dedicated to the discovery of unexpected and/or emergent viruses in blood products or donors - a strategy more suitable for epidemiological watch studies, rather than a defined viral detection in blood products. With several filters to remove unspecific and/or low significant signals, the ezVIR diagnostics-oriented pipeline was described by Cordey et al. and colleagues $[2,31,32]$ and specifically used for viral screening in RBCU, FFP [2] and PC [18]. Several other diagnostics-oriented pipelines have also been described [33]. Designed for routine use in clinical laboratories, these pipelines have the advantage of including many filters for quick turnaround reporting without the need for timeconsuming analysis. However, the use of more or less stringent filters in these pipelines has the risk of low specificity and overlooking unexpected signatures. In parallel with another group from the Blood Systems Research Institute (San Francisco), we compared results from an analysis of 300 Swiss PC using the ezVIR (diagnostics-oriented) and VIRGO (virus discovery-oriented) pipelines. Interestingly, even when considering the differences in analysis pipelines, both showed the same conclusions with respect to viral landscape (unpubl. observations).

\section{Technical Advantages and Limitations of NGS Applied to Blood Products and Donors}

NGS is a unique tool that allows for complete sequencing of viral nucleic acids. It represents the ideal approach to screen for unexpected or emerging viruses in blood donors, blood products or defined populations with a higher risk of viral infection. However, it is not suitable to individually determine the sensitivity of detection of all referenced viruses. Thus, NGS is likely to never validate the absence of risk in the case of a negative screen, i.e. false-negative reporting. Moreover, NGS does not provide adequate information relating to the depth/percent of genome coverage to definitively report about the presence (or not) of a viral signature. This means that an individual threshold for each virus, validated by the appropriate diagnostic pipeline, would be necessary to ensure the absence of risk in the event of a negative screen. It is thus considered, and currently accepted, that NGS could overlook some viruses, and that it is a rather useful tool to determine the presence of unexpected/emergent viruses in blood banks. To estimate the possibility of virus overlooking by NGS, our group reported experiments comparing NGS and conventional NAT for the detection of TTV, suggesting that NAT is more sensitive [2]. Further limitations of NGS in the field of transfusion medicine include: (i) the risk of misattributions of reads sharing sequence homology with the human genome or between different virus strains (as previously mentioned), (ii) the need to control for environmental contaminants in NGS instruments and reagents, (iii) the frequent need of a pooling strategy to reduce costs.

\section{Clinical Interpretation of NGS Data in Donors and Blood Products}

Considering the mass of data generated by NGS approaches, the analysis is far more complicated when compared to conventional NAT. This is a real and significant challenge for laboratories, requiring the need for specialized expertise. The identification of GBM in donors and recipient's plasma, later attributed to a bench contamination, well illustrates the difficulties in data interpretation. The first reported identification of viral signatures by NGS in individuals raised quite some concern in the blood transfusion community. In clinical practice, if NGS technology becomes more widely used in medicine, transfusion specialists and clinicians will have to face new challenges in terms of data interpretation and decisions. They will have to deal with a multitude of identified viruses, more or less known, both in blood donors and products. They will have to provide a clear and validated strategy for interpretation, based on available evidence and use of appropriate controls. Being aware of the presence of viruses in donors prior to screening would significantly reduce the need for interpretation of confounding viral sequences, which is also pertinent when it comes to explaining a clinical event or documented transfusion-transmitted infection. This approach would need to be carefully managed to avoid it being a double-edged sword in that any restrictive measure based on precautionary principles could threaten self-sufficiency of blood supply. It is thus essential that carefully constructed guidelines and good practices in transfusion medicine be implemented, which takes into account the risk of transfusion-transmitted infection, the disease concerned, the patient, the product characteristics and viral load.

\section{Epidemiology and Clinical Features of the Disease}

Seroprevalence of a viral disease is geographically variable but should be comparable between blood donors and recipients living in the same region. A previous immuni- 
zation should mitigate the risk of recipient contamination. However, it is difficult to quantitatively estimate the protective effect of a previous immunization in both the recipient and the donor. It is also important to balance the environmental risk of transmission with the risk of transmission by transfusion. The severity of a viral disease is estimated by a specific mortality and morbidity, but also by our ability to prevent and treat the infection. For example, the transmission of hepatitis $\mathrm{E}$ virus by transfusion has been demonstrated, but data to estimate the clinical impact on recipients are limited. There is no vaccine but a treatment is available [34]. Although sensible, finding the best strategy between screening all donors and closely monitoring recipients may not be feasible.

\section{Clinical State of the Recipient}

The health condition of a patient exposed to a viral contaminant by transfusion is directly related to the risk of developing a clinically relevant disease. This is not limited to the level of immunosuppression but also to other factors such as the viral species implicated, medical history, pregnancy (or stage of pregnancy), hemolysis, marrow failure or liver disease.

\section{Fraction and Manufacturing of Blood Components}

The fraction of blood being manufactured and transfused also has an effect on virus transmission rate. In blood components, the content of significant amounts of donor antibodies (such as in FFP, PC) could reduce the risk of transmission. Parvovirus B19 is well known for its risk of transmission from pooled plasma infusion, but to a lesser extent from other individual blood components [35]. Leukoreduction and pathogen inactivation should also be taken into account.

\section{Viral Load}

The level of viremia is directly correlated with the risk of transfusion-transmission. A critical threshold should exist for each virus. Concerning common viruses like the parvovirus B19, enough data were available for regulators to suggest an appropriate threshold of less than or equal to $10^{4} \mathrm{IU} / \mathrm{mL}$ for plasma donation [35]. To date, there is no validated method available for NGS to provide a standardized quantification.

\section{Conclusion}

NGS offers the possibility of screening for a large range of viral signatures and hence is the ideal tool to discover potentially dangerous and/or emergent viruses in donor populations. As the costs and technical expertise in this domain become more favorable, the implementation of NGS into routine diagnostic practices will increase. However, considering the complexity of clinical interpretation, reaching consensus between the professionals in the field of blood transfusion will remain a challenge and a critical aspect to address. Even with a lack of data, experts in transfusion medicine should collectively define the most appropriate screening policies for blood products.

\section{Disclosure Statement}

The authors declare that they have no conflict of interest for the publication of this review.

\section{References}

1 Williams SH, Cordey S, Bhuva N, Laubscher F, Hartley MA, Boillat-Blanco N, et al. Investigation of the plasma virome from cases of unexplained febrile illness in Tanzania from 2013 to 2014: a comparative analysis between unbiased and VirCapSeq-VERT highthroughput sequencing approaches. MSphere. 2018 Aug;3(4):e00311-18.

2 Lau P, Cordey S, Brito F, Tirefort D, Petty TJ Turin L, et al. Metagenomics analysis of red blood cell and fresh-frozen plasma units. Transfusion. 2017 Jul;57(7):1787-800.

3 Janiak M, Caraballo Cortés K, Perlejewski K, Kubicka-Russel D, Grabarczyk P, Demkow U, et al. Next-generation sequencing of hepatitis $\mathrm{C}$ virus (HCV) mixed-genotype infections in anti-HCV-negative blood donors. Adv Exp Med Biol. 2018;1096:65-71.
4 Moustafa A, Xie C, Kirkness E, Biggs W, Wong E, Turpaz Y, et al. The blood DNA virome in 8,000 humans. PLoS Pathog. 2017 Mar;13(3):e1006292.

5 Breitbart M, Rohwer F. Method for discovering novel DNA viruses in blood using viral particle selection and shotgun sequencing. Biotechniques. 2005 Nov;39(5):729-36.

6 Furuta RA, Sakamoto H, Kuroishi A, Yasiui K, Matsukura H, Hirayama F. Metagenomic profiling of the viromes of plasma collected from blood donors with elevated serum alanine aminotransferase levels. Transfusion. 2015 Aug;55(8):1889-99.

7 Focosi D, Antonelli G, Pistello M, Maggi F. Torquetenovirus: the human virome from bench to bedside. Clin Microbiol Infect. 2016 Jul;22(7):589-93.
8 Rascovan N, Duraisamy R, Desnues C. Metagenomics and the Human Virome in Asymptomatic Individuals. Annu Rev Microbiol. 2016 Sep;70(1):125-41.

9 Pancaldi C, Corazzari V, Maniero S, Mazzoni E, Comar M, Martini F, et al. Merkel cell polyomavirus DNA sequences in the buffy coats of healthy blood donors. Blood. 2011 Jun; 117(26):7099-101.

10 Sauvage V, Laperche S, Cheval J, Muth E, Dubois M, Boizeau L, et al. Viral metagenomics applied to blood donors and recipients at high risk for blood-borne infections. Blood Transfus. 2016 Sep;14(5):400-7.

11 Šroller V, Hamšíková E, Ludvíková V, Musil J, Němečková Š, Saláková M. Seroprevalence rates of HPyV6, HPyV7, TSPyV, HPyV9, MWPyV and KIPyV polyomaviruses among the healthy blood donors. J Med Virol. 2016 Jul;88(7):1254-61. 
12 Urbano PR, Nali LH, Bicalho CS, Pierrotti LC, David-Neto E, Pannuti CS, et al. New findings about trichodysplasia spinulosa-associated polyomavirus (TSPyV) - novel qPCR detects TSPyV-DNA in blood samples. Diagn Microbiol Infect Dis. 2016 Feb;84(2):123-4.

13 Popgeorgiev N, Boyer M, Fancello L, Monteil S, Robert C, Rivet R, et al. Marseillevirus-like virus recovered from blood donated by asymptomatic humans. J Infect Dis. 2013 Oct; 208(7):1042-50.

14 Popgeorgiev N, Colson P, Thuret I, Chiarioni J, Gallian P, Raoult D, et al. Marseillevirus prevalence in multitransfused patients suggests blood transmission. J Clin Virol. 2013 Dec;58(4):722-5.

15 Phan TG, Desnues C, Switzer WM, Djoko CF, Schneider BS, Deng X, et al. Absence of giant blood Marseille-like virus DNA detection by polymerase chain reaction in plasma from healthy US blood donors and serum from multiply transfused patients from Cameroon. Transfusion. 2015 Jun;55(6):1256-62.

16 Sauvage V, Livartowski A, Boizeau L, ServantDelmas A, Lionnet F, Lefrère JJ, et al. No evidence of Marseillevirus-like virus presence in blood donors and recipients of multiple blood transfusions. J Infect Dis. 2014 Dec;210(12): 2017-8.

17 Chen AC, Keleher A, Kedda MA, Spurdle AB McMillan NA, Antonsson A. Human papillomavirus DNA detected in peripheral blood samples from healthy Australian male blood donors. J Med Virol. 2009 Oct;81(10):1792-6.

18 Brito F, Cordey S, Delwart E, Deng X, Tirefort D, Lemoine-Chaduc C, et al. Metagenomics analysis of the virome of 300 concentrates from a Swiss platelet bank. Vox Sang. 2018 Jul; 113(6):601-4.

19 Zhang W, Li L, Deng X, Blümel J, Nübling CM, Hunfeld A, et al. Viral nucleic acids in human plasma pools. Transfusion. 2016 Sep; 56(9):2248-55
20 Krupovic M, Ghabrial SA, Jiang D, Varsani A. Genomoviridae: a new family of widespread single-stranded DNA viruses. Arch Virol. 2016 Sep;161(9):2633-43.

21 Meyer TJ, Rosenkrantz JL, Carbone L, Chavez SL. Endogenous retroviruses: with us and against us. Front Chem. 2017 Apr;5:23.

22 Anbarlou A, AkhavanRahnama M, Atashi A, Soleimani M, Arefian E, Gallinella G. Possible involvement of miRNAs in tropism of Parvovirus B19. Mol Biol Rep. 2016 Mar;43(3):17581.

23 Attatippaholkun N, Kosaisawe N, U-Pratya Y, Supraditaporn P, Lorthongpanich C, Pattanapanyasat $\mathrm{K}$, et al. Selective tropism of dengue virus for human glycoprotein Ib. Sci Rep. 2018 Feb;8(1):2688.

24 Ariede JR, Pardini MI, Silva GF, Grotto RM. Platelets can be a biological compartment for the hepatitis $\mathrm{C}$ virus. Braz J Microbiol. 2015 Jun;46(2):627-9.

25 Stewart CM, Tsui DW. Circulating cell-free DNA for non-invasive cancer management. Cancer Genet. 2018 Dec;228-229:169-79.

26 Zaporozhchenko IA, Ponomaryova AA, Rykova EY, Laktionov PP. The potential of circulating cell-free RNA as a cancer biomarker: challenges and opportunities. Expert Rev Mol Diagn. 2018 Feb;18(2):133-45.

27 Waldvogel Abramowski S, Tirefort D, Lau P, Guichebaron A, Taleb S, Modoux C, et al. Cell-free nucleic acids are present in blood products and regulate genes of innate immune response. Transfusion. 2018 Jul;58(7): 1671-81.

28 Naccache SN, Greninger AL, Lee D, Coffey LL, Phan T, Rein-Weston A, et al. The perils of pathogen discovery: origin of a novel parvovirus-like hybrid genome traced to nucleic acid extraction spin columns. J Virol. 2013 Nov;87(22):11966-77.

29 Ngoi CN, Siqueira J, Li L, Deng X, Mugo P, Graham SM, et al. The plasma virome of febrile adult Kenyans shows frequent parvovirus B19 infections and a novel arbovirus (Kadipiro virus). J Gen Virol. 2016 Dec;97(12): 3359-67.
30 Simmons G, Glynn SA, Komaroff AL, Mikovits JA, Tobler LH, Hackett J Jr, et al.; Blood XMRV Scientific Research Working Group (SRWG). Failure to confirm XMRV/MLVs in the blood of patients with chronic fatigue syndrome: a multi-laboratory study. Science. 2011 Nov;334(6057):814-7.

31 Cordey S, Bel M, Petty TJ, Docquier M, Sacco $\mathrm{L}$, Turin L, et al. Toscana virus meningitis case in Switzerland: an example of the ezVIR bioinformatics pipeline utility for the identification of emerging viruses. Clin Microbiol Infect. 2015 Apr;21(4):387 e1-4.

32 Petty TJ, Cordey S, Padioleau I, Docquier M, Turin L, Preynat-Seauve O, et al. Comprehensive human virus screening using highthroughput sequencing with a user-friendly representation of bioinformatics analysis: a pilot study. J Clin Microbiol. 2014 Sep;52(9): 3351-61.

33 Nooij S, Schmitz D, Vennema H, Kroneman A, Koopmans MP. Overview of virus metagenomic classification methods and their biological applications. Front Microbiol. 2018 Apr;9:749.

34 Dalton HR, Kamar N, Baylis SA, Moradpour D, Wedemeyer H, Negro F; European Association for the Study of the Liver. Electronic address: easloffice@easloffice.eu; European Association for the Study of the Liver. EASL Clinical Practice Guidelines on hepatitis E virus infection. J Hepatol. 2018 Jun;68(6):125671.

35 Kleinman SH, Glynn SA, Lee TH, Tobler LH, Schlumpf KS, Todd DS, et al.; National Heart, Lung, and Blood Institute Retrovirus Epidemiology Donor Study-II (NHLBI REDS-II). A linked donor-recipient study to evaluate parvovirus B19 transmission by blood component transfusion. Blood. 2009 Oct;114(17): 3677-83. 